\title{
An Algebraic and Graph Theoretic Framework to Study Monomial Dynamical Systems over a Finite Field
}

\author{
Edgar Delgado-Eckert
}

ETH Zürich, Department of Biosystems Science and Engineering (D-BSSE) WRO-1058-8.46, Mattenstrasse 26, 4058 Basel, Switzerland edgar.delgado-eckert@mytum.de

\begin{abstract}
A monomial dynamical system $f: K^{n} \rightarrow K^{n}$ over a finite field $K$ is a nonlinear deterministic time discrete dynamical system with the property that each component function $f_{i}: K^{n} \rightarrow K$ is a monic nonzero monomial function. In this paper we provide an algebraic and graph theoretic framework to study the dynamic properties of monomial dynamical systems over a finite field. Within this framework, characterization theorems for fixed point systems, that is, systems in which all trajectories end in steady states, are proved. These characterizations are stated in terms of connectedness properties of the dependency graph. Our formalism allowed us to develop an algorithm of polynomial complexity for testing whether or not a given monomial dynamical system over an arbitrary finite field is a fixed point system. In addition, we were able to identify a class of monomial dynamical systems, namely, the $(q-1)$ fold redundant monomial systems. Within this class of systems a characterization of fixed point systems is proved that represents a generalization of previous work on Boolean monomial dynamical systems.
\end{abstract}

\section{Introduction}

Time discrete dynamical systems over a finite set $X$ are an important subject of active mathematical research. One relevant example of such systems are cellular automata, first introduced in the late 1940s by John von Neumann [1]. More general examples are nondeterministic finite state automata [2] and sequential dynamical systems [3].

Deterministic time discrete dynamical systems over a finite field are mappings $f: K^{n} \rightarrow K^{n}$, where $K$ is a finite field and $n \in \mathbb{N}$ is the dimension of the system. As opposed to deterministic time discrete dynamical systems over a finite set $X$, this type of system allows for a richer mathematical framework within which they can be studied. For instance, it can be shown that every component function $f_{i}: K^{n} \rightarrow K$ is a polynomial function of bounded degree in $n$ variables $[4,5]$.

The study of dynamical systems generally addresses the question of the system's long-term behavior, in particular, the existence of fixed 
points and (limit) cyclic trajectories. In this paper we provide an algebraic and graph theoretic framework for studying a specific class of nonlinear time discrete dynamical systems over a finite field, namely, monomial dynamical systems over a finite field. In such systems, every component function $f_{i}: K^{n} \rightarrow K$ is a monic nonzero monomial function.

Some types of monomial systems and their dynamic behavior have been studied before: monomial cellular automata [6, 7], Boolean monomial systems [8], monomial systems over the $p$-adic numbers [9, 10], and monomial systems over a finite field [11-13]. A necessary and sufficient condition for Boolean monomial systems to be fixed point systems, that is, systems in which all trajectories end in steady states, is proved in [8]. This condition could be algorithmically exploited. Indeed, the authors make some suggestive comments in that direction [8, Section 4.3]. Moreover, they describe the structure of the limit cycles of a special type of Boolean monomial systems.

In [13] the authors present a necessary and sufficient condition for monomial systems over a finite field to be fixed point systems. However, this condition is not easily verifiable and therefore the theorem does not yield a tractable algorithm in a straightforward way. In [13] it is explicitly stated that a tractable algorithm to determine whether or not a monomial dynamical system over an arbitrary finite field is a fixed point system has still to be developed [13, Section 3]. Nevertheless, while the present paper was being peer-reviewed, [14] was published, providing the missing computational-algebraic ingredient toward an algorithmic application of the approach in [13]. However, the concrete details of an algorithm exploiting the results in [13] and [14] still need to be developed.

Our work was influenced by $[8,13,12]$. However, we took a slightly different approach. The mathematical formalism we developed allows for a deeper understanding of monomial dynamical systems over a finite field. Indeed, we were able to circumvent the complicated Glueing-procedure developed in [8, Section 5]. Our formalism allows formulating a very simple algorithm of polynomial complexity for testing whether or not a given monomial dynamical system over an arbitrary finite field is a fixed point system. Additional theorems that complement the work in $[8,13]$ are obtained. Furthermore, we were able to identify a class of monomial dynamical systems, namely, the $(q-1)$-fold redundant monomial systems (to be defined later). Within this class of systems, a very satisfying characterization of fixed point systems can be reached. Boolean systems are trivial examples of $(q-1)$-fold redundant systems. As a consequence, many of our results about $(q-1)$-fold redundant monomial systems provide a generalization of theorems proved in [8] for Boolean systems. Last but not least, our formalism also constitutes a basis for the study of monomial control systems [15]. 
It is pertinent to mention the work of [16] regarding linear time discrete dynamical systems over a finite field, in which the number of limit cycles and their lengths is linked to the factorization (in so-called elementary divisor polynomials) of the characteristic polynomial of the matrix representing the system. (See also [17] for a more mathematical exposition and $[2,18]$ for applications of the Boolean case in control theory.) Furthermore, the affine case, that is, a linear map followed by a translation, was studied in [19]. An interesting contribution was made by Paul Cull in [20], who extended the considerations to nonlinear functions, and showed how to reduce them to the linear case. However, Cull's approach does not yield an algorithm of polynomial complexity to solve the steady state system problem. Moreover, according to [21], this might in general not be possible as a matter of principle.

This paper is organized as follows: Section 2 establishes an algebraic and graph theoretic framework within which monomial dynamical systems over a finite field are studied. It starts with some basic definitions and algebraic results (proofs can be found in [5, Section 2.2]) and leads the reader to the first important result, namely, that the monoid of $n$-dimensional monomial dynamical systems over a finite field $K$ is isomorphic to a certain monoid of matrices. Section 2 finishes with propositions about the relationship between the matrix $F$ corresponding to a monomial system $f$ (via the isomorphism mentioned) and the adjacency matrix of the dependency graph of $f$. These findings allow us to link topological properties of the dependency graph with the dynamics of $f$, which is the fundamental insight underlying this work.

Section 3 is devoted to the characterization of fixed point systems. These characterizations are stated in terms of connectedness properties of the dependency graph. Theorem 4, for instance, presents necessary and sufficient connectedness conditions for a system to be a fixed point system. Furthermore, the class of $(q-1)$-fold redundant monomial systems is introduced and a characterization of fixed point systems within this class is provided. We finish Section 3 with several relatively easy to test sufficient conditions for a system to be a fixed point system.

Section 4 presents an algorithm of polynomial complexity to test whether or not a given monomial dynamical system over a finite field $K$ is a fixed point system. A detailed complexity analysis of the algorithm is provided. 


\section{Algebraic and Graph Theoretic Formalism}

In this section we introduce the monoid of $n$-dimensional monomial dynamical systems over a finite field $\mathrm{F}_{q}$. This monoid is isomorphic to a certain monoid of matrices; in other words, the composition $f \circ g$ of two monomial dynamical systems $f, g$ is completely captured by the product $F \cdot G$ of their corresponding matrices. Furthermore, we provide our definition for the dependency graph of a monomial dynamical system $f$. The adjacency matrix of the dependency graph is precisely the matrix $F$ associated with $f$ via the isomorphism mentioned earlier. This finding allows us to link topological properties of the dependency graph with the dynamics of $f$, which is the fundamental insight underlying this work.

Definition 1. We denote a finite field with $\mathbf{F}_{q}$, where $q$ stands for the number of elements in the field. It is understood that $q$ is a power of the (prime) characteristic of the field.

Definition 2. Let $\mathbf{F}_{q}$ be a finite field. The set $E_{q}:=\{0, \ldots, q-1\} \subset \mathbb{N}_{0}$ is called the exponents set of the field $\mathrm{F}_{q}$.

Definition 3. Let $\mathrm{F}_{q}$ be a finite field and $n \in \mathbb{N}$. A map $f: \mathbf{F}_{q}^{n} \rightarrow \mathbf{F}_{q}^{n}$ is called a monomial dynamical system over $\mathrm{F}_{q}$ if for every $i \in\{1, \ldots, n\}$ there exists a tuple $\left(F_{i 1}, \ldots, F_{i n}\right) \in E_{q}^{n}$ such that $f_{i}(x)=x_{1}^{F_{i 1}} \cdots x_{n}^{F_{i n}} \forall x \in \mathrm{F}_{q}^{n}$.

Remark 1. As opposed to [8], we exclude in the definition of a monomial dynamical system the possibility that one of the functions $f_{i}$ is equal to the zero function. However, in contrast to [13], we do allow the case $f_{i} \equiv 1$ in our definition. This is not a loss of generality because of the following: If we were studying a dynamical system $f: \mathbf{F}_{q}^{n} \rightarrow \mathbf{F}_{q}^{n}$ where one of the functions, say $f_{j}$, was equal to zero, then, for every initial state $x \in \mathrm{F}_{q}^{n}$, after one iteration the system would be in a state $f(x)$ whose $j^{\text {th }}$ entry is zero. In all subsequent iterations the value of the $j^{\text {th }}$ entry would remain zero. As a consequence, the longterm dynamics of the system are reflected in the projection $\pi_{\hat{j}}(y):=\left(y_{1}, \ldots, y_{j-1}, y_{j+1}, \ldots, y_{n}\right)^{t}$ and it is sufficient to study the system

Complex Systems, 18 ㄷ 2009 Complex Systems Publications, Inc. 


$$
\begin{aligned}
& \tilde{f}: \mathbf{F}_{q}^{n-1} \rightarrow \mathbf{F}_{q}^{n-1} \\
& y \mapsto\left(\begin{array}{c}
f_{1}\left(y_{1}, \ldots, y_{j-1}, 0, y_{j+1}, \ldots, y_{n}\right) \\
\vdots \\
f_{j-1}\left(y_{1}, \ldots, y_{j-1}, 0, y_{j+1}, \ldots, y_{n}\right) \\
f_{j+1}\left(y_{1}, \ldots, y_{j-1}, 0, y_{j+1}, \ldots, y_{n}\right) \\
\vdots \\
f_{n}\left(y_{1}, \ldots, y_{j-1}, 0, y_{j+1}, \ldots, y_{n}\right)
\end{array}\right) .
\end{aligned}
$$

In general, the system $\tilde{f}$ could contain component functions equal to the zero function, since every component $f_{i}$ that depends on the variable $x_{j}$ would become zero. As a consequence, the described procedure needs to be applied several times until the lower dimensional system obtained does not contain component functions equal to zero. It is also possible that this repeated procedure yields the onedimensional zero function. In this case, we can conclude that the original system $f$ is a fixed point system with $(0, \ldots, 0) \in \mathrm{F}_{q}^{n}$ as its unique fixed point. The details about this procedure are described as the "preprocessing algorithm" in [5, Appendix B].

It is well known that every function $h: \mathrm{F}_{q}^{n} \rightarrow \mathrm{F}_{q}$ is a polynomial function in $n$ variables where no variable appears to a power higher or equal to $q$ (e.g., [4, pp. 368-369]; or [5, Section 1.2]). Calculating the composition of a dynamical system $f: \mathbf{F}_{q}^{n} \rightarrow \mathbf{F}_{q}^{n}$ with itself, we face the situation where some of the exponents exceed the value $q-1$ and need to be reduced according to the well-known rule

$$
a^{q}=a \forall a \in \mathrm{F}_{q} .
$$

This process can be accomplished systematically if we look at the power $x_{i}^{p}$ (where $p>q$ ) as a polynomial in the ring $\mathrm{F}_{q}[\tau]$ and define the magnitude $\operatorname{red}_{q}(p)$ as the degree of the (unique) remainder of the polynomial division $\tau^{p} \div\left(\tau^{q}-\tau\right)$ in the polynomial ring $\mathrm{F}_{q}[\tau]$. Then we can write

$$
x_{i}^{p}=x_{i}^{\operatorname{red}_{q}(p)} \forall x_{i} \in \mathrm{F}_{q},
$$

which is a direct consequence of the following easily shown properties (e.g., [5, Lemma 39]) of the operator $\operatorname{red}_{q}: \mathbb{N}_{0} \rightarrow E_{q}$ :

$$
\operatorname{red}_{q}\left(\operatorname{red}_{q}(c)\right)=\operatorname{red}_{q}(c) \forall c \in \mathbb{N}_{0}
$$




$$
\begin{aligned}
& \operatorname{red}_{q}(c)=0 \Leftrightarrow c=0 \\
& \text { for } a, b \in \mathbb{N}_{0}, x^{a}=x^{b} \forall x \in \mathbf{F}_{q} \Leftrightarrow \operatorname{red}_{q}(a)=\operatorname{red}_{q}(b) \\
& \text { for } a, b \in \mathbb{N}, \\
& \operatorname{red}_{q}(a)=\operatorname{red}_{q}(b) \Leftrightarrow \exists \alpha \in \mathbb{Z}: a=b+\alpha(q-1) .
\end{aligned}
$$

In conclusion, the "exponents arithmetic" needed when calculating the composition of dynamical systems $f, g: \mathrm{F}_{q}^{n} \rightarrow \mathrm{F}_{q}^{n}$ can be formalized based on the reduction operator $\operatorname{red}_{q}(p)$. Indeed, the set $E_{q}=\{0,1, \ldots,(q-1)\} \subset \mathbb{Z}$ together with the operations of addition $a \oplus b:=\operatorname{red}_{q}(a+b)$ and multiplication $\mathrm{a} \bullet \mathrm{b}:=\operatorname{red}_{q}(a b)$ is a commutative semiring with identity 1 . We call this commutative semiring the exponents semiring of the field $\mathrm{F}_{q}$. With this operation, it can be easily shown that the set $M\left(n \times n ; E_{q}\right)$ of $n \times n$ quadratic matrices with entries in the semiring $E_{q}$ together with the operation . of matrix multiplication (which is defined in terms of the operations $\oplus$ and $\bullet$ on the matrix entries) over $E_{q}$ is a monoid. Similarly, the set

$$
\begin{aligned}
M F_{n}^{n}\left(\mathbf{F}_{q}\right):= & \left\{f: \mathbf{F}_{q}^{n} \rightarrow \mathbf{F}_{q}^{n} \mid\right. \\
& \left.\exists F \in M\left(n \times n ; E_{q}\right): f_{i}(x)=x_{1}^{F_{i 1}} \cdots x_{n}^{F_{i n}} \forall x \in \mathbf{F}_{q}^{n}\right\}
\end{aligned}
$$

of all $n$-dimensional monomial dynamical systems over $\mathrm{F}_{q}$ together with the composition o of mappings is a monoid. It is not hard to prove that these two monoids are isomorphic via the isomorphism

$$
\begin{aligned}
\Psi & : M\left(n \times n ; E_{q}\right) \rightarrow M F_{n}^{n}\left(\mathbf{F}_{q}\right) \\
G & \mapsto \Psi(G)
\end{aligned}
$$

where

$$
\Psi(G)_{i}(x):=x_{1}^{G_{i 1}} \ldots x_{n}^{G_{i n}} \text { for } i=1, \ldots, n .
$$

The interested reader can find proofs for the previous results in $[5$, Section 2.2].

Remark 2. The operation $\operatorname{red}_{q}: \mathbb{N}_{0} \rightarrow E_{q}$ can be extended to matrices $M\left(n \times n ; \mathbb{N}_{0}\right)$ by applying $\operatorname{red}_{q}$ to the entries of the matrix. We call this extension $\operatorname{mred}_{q}: M\left(n \times n ; \mathbb{N}_{0}\right) \rightarrow M\left(n \times n ; E_{q}\right)$. See [5, Remark 47] for further details where one important property of $\operatorname{mred}_{q}$ shown is

$$
\operatorname{mred}_{q}(A)=0 \Leftrightarrow A=0 .
$$


Remark 3. For a given monomial dynamical system $f \in M F_{n}^{n}\left(\mathbf{F}_{q}\right)$, the matrix $F:=\Psi^{-1}(f)$ is called the corresponding matrix of the system $f$. For a matrix power in the monoid $M\left(n \times n ; E_{q}\right)$, we use the notation $F^{\cdot m}$. By induction it can be easily shown that $\Psi^{-1}\left(f^{m}\right)=F^{\cdot m}$.

As our investigations have shown, topological properties of the dependency graph of a monomial dynamical system (defined later) can reveal dynamic properties of the system. Therefore, in the rest of this section we turn our attention to some graph theoretic considerations. We now review some well-known graph theoretical objects and results while, at the same time, we introduce some useful notation.

Definition 4. A directed graph $G=\left(V_{G}, E_{G}, \pi_{G}: E_{G} \rightarrow V_{G} \times V_{G}\right)$ that allows self loops and parallel directed edges is called a digraph.

Definition 5. Let $M$ be a nonempty finite set. Furthermore, let $n:=|M|$ be the cardinality of $M$. An enumeration of the elements of $M$ is a bijective mapping $f: M \rightarrow\{1, \ldots, n\}$. Given an enumeration $f$ of the set $M$, we write $M=\left\{f_{1}, \ldots, f_{n}\right\}$ where the unique element $x \in M$ with the property $f(x)=i \in\{1, \ldots, n\}$ is denoted as $f_{i}$.

Definition 6. Let $f \in M F_{n}^{n}\left(\mathrm{~F}_{q}\right)$ be a monomial dynamical system and $G=\left(V_{G}, E_{G}, \pi_{G}\right)$ a digraph with vertex set $V_{G}$ of cardinality $\left|V_{G}\right|=n$. Furthermore, let $F:=\Psi^{-1}(f)$ be the corresponding matrix of $f$. The digraph $G$ is called a dependency graph of $f$ if and only if an enumeration $a: V_{G} \rightarrow\{1, \ldots, n\}$ of the elements of $V_{G}$ exists, such that $\forall i, j \in\{1, \ldots, n\}$ there are exactly $F_{i j}$ directed edges $a_{i} \rightarrow a_{j}$ in the set $E_{G}$, that is, $\left|\pi_{f}^{-1}\left(\left(a_{i}, a_{j}\right)\right)=F_{i j}\right|$.

Remark 4. It is easy to show that if $G$ and $H$ are dependency graphs of $f$, then $G$ and $H$ are isomorphic. In this sense we speak of the dependency graph of $f$ and denote it by $G_{f}=\left(V_{f}, E_{f}, \pi_{f}\right)$. Note that our definition of a dependency graph differs slightly from the definition used in [8].

Definition 7. Let $G=\left(V_{G}, E_{G}, \pi_{G}\right)$ be a digraph. Two vertices $a, b \in V_{G}$ are called connected if there is a $t \in \mathbb{N}_{0}$ and (not necessarily different) vertices $v_{1}, \ldots, v_{t} \in V_{G}$ such that $a \rightarrow v_{1} \rightarrow v_{2} \rightarrow \cdots$ $\rightarrow v_{t} \rightarrow b$. In this situation we write $a \rightarrow_{s} b$, where $s$ is the number of directed edges involved in the sequence from $a$ to $b$ (in this case $s=t+1)$. Two sequences $a \rightarrow_{s} b$ of the same length are considered different if the directed edges involved are different or the order at which they appear is different, even if the visited vertices are the same. As a convention, a single vertex $a \in V_{G}$ is always connected to itself $a \rightarrow_{0} a$ by an empty sequence of length 0 . 
Definition 8. Let $G=\left(V_{G}, E_{G}, \pi_{G}\right)$ be a digraph and $a, b \in V_{G}$ two vertices. A sequence $a \rightarrow_{s} b$

$$
a \rightarrow v_{1} \rightarrow v_{2} \rightarrow \cdots \rightarrow v_{t} \rightarrow b
$$

is called a path, if no vertex $v_{i}$ is visited more than once. If $a=b$, but no other vertex is visited more than once, $a \rightarrow_{s} b$ is called a closed path.

Definition 9. Let $G=\left(V_{G}, E_{G}, \pi_{G}\right)$ be a digraph. Two vertices $a, b \in V_{G}$ are called strongly connected if there are natural numbers $s, t \in \mathbb{N}$ such that $a \rightarrow_{s} b$ and $b \rightarrow_{t} a$. In this situation we write $a \rightleftharpoons b$.

Theorem 1. Let $G=\left(V_{G}, E_{G}, \pi_{G}\right)$ be a digraph. $\rightleftharpoons$ is an equivalence relation on $V_{G}$ called a strong equivalence. The equivalence class of any vertex $a \in V_{G}$ is called a strongly connected component and denoted

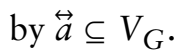

Proof. This is well known as shown, for instance, in [8, Definition 3.1].

Definition 10. Let $G=\left(V_{G}, E_{G}, \pi_{G}\right)$ be a digraph and $a \in V_{G}$ one of its vertices. The strongly connected component $\stackrel{\leftrightarrow}{a} \subseteq V_{G}$ is called trivial if and only if $\stackrel{a}{a}=\{a\}$ and there is no edge $a \rightarrow a$ in $E_{G}$.

Definition 11. Let $G=\left(V_{G}, E_{G}, \pi_{G}\right)$ be a digraph with vertex set $V_{G}$ of cardinality $\left|V_{G}\right|=n$ and $V_{G}=\left\{a_{1}, \ldots, a_{n}\right\}$ an enumeration of the elements of $V_{G}$. The matrix $A \in M\left(n \times n ; \mathbb{N}_{0}\right)$ whose entries are defined as $A_{i j}:=$ number of edges $a_{i} \rightarrow a_{j}$ contained in $E_{G}$ for $i, j=1, \ldots, n$ is called an adjacency matrix of $G$ with the enumeration $a$.

Theorem 2. Let $G=\left(V_{G}, E_{G}, \pi_{G}\right)$ be a digraph with vertex set $V_{G}$ of cardinality $\left|V_{G}\right|=n$ and $V_{G}=\left\{a_{1}, \ldots, a_{n}\right\}$ an enumeration of the elements of $V_{G}$. Furthermore, let $A \in M\left(n \times n ; \mathbb{N}_{0}\right)$ be its adjacency matrix (with the enumeration $a$ ), $m \in \mathbb{N}$ a natural number, and $B:=A^{m} \in M\left(n \times n ; \mathbb{N}_{0}\right)$ is the $m^{\text {th }}$ power of $A$. Then $\forall i, j \in\{1, \ldots, n\}$ the entry $B_{i j}$ of $B$ is equal to the number of different sequences $a_{i} \rightarrow m a_{j}$ of length $m$.

Proof. The proof of this is a well-known result and can be found in [22].

Remark 5. Let $f \in M F_{n}^{n}\left(\mathrm{~F}_{q}\right)$ be a monomial dynamical system. Furthermore, let $G_{f}=\left(V_{f}, E_{f}, \pi_{f}\right)$ be the dependency graph of $f$ and $V_{f}=\left\{a_{1}, \ldots, a_{n}\right\}$ the associated enumeration of the elements of $V_{f}$. Then, according to the definition of a dependency graph, $F:=\Psi^{-1}(f)$ 
(the corresponding matrix of $f$ ) is precisely the adjacency matrix of $G_{f}$ with the enumeration $a$. Now, by Remarks 3 and 2 we can conclude

$$
\Psi^{-1}\left(f^{m}\right)=\operatorname{mred}_{q}\left(F^{m}\right) .
$$

\section{Characterization of Fixed Point Systems}

The results presented in Section 2 allow us to link topological properties of the dependency graph with the dynamics of $f$. We now exploit this feature to prove some characterizations of fixed point systems stated in terms of connectedness properties of the dependency graph. In the course of these investigations we will identify and define a class of monomial dynamical systems as the $(q-1)$-fold redundant monomial systems. Within this class of systems, a very satisfying characterization of fixed point systems can be reached. A trivial example of $(q-1)$-fold redundant systems are the Boolean systems, that is, monomial systems $f \in M F_{n}^{n}\left(F_{2}\right)$. As a consequence, many of the results shown in this section provide a generalization of results proved in [8] for Boolean systems.

Theorem 3. Let $\mathrm{F}_{q}$ be a finite field and $f \in M F_{n}^{n}\left(\mathrm{~F}_{q}\right)$ a monomial dynamical system. Then $f$ is a fixed point system with $(1, \ldots, 1)^{t} \in \mathrm{F}_{q}^{n}$ as its only fixed point if and only if its dependency graph only contains trivial strongly connected components.

Proof. By Remark 5, $F:=\Psi^{-1}(f)$ is the adjacency matrix of the dependency graph of $f$. If the dependency graph does not contain any nontrivial strongly connected components, every sequence $a \rightarrow_{s} b$ between two arbitrary vertices can be at most of length $n-1$. (A sequence that revisits a vertex would contain a closed sequence, which is strongly connected.) Therefore, by Theorem 2, $\exists m \in \mathbb{N}$ with $m \leq n$ such that $F^{m}=0$ (the zero matrix in $M\left(n \times n ; \mathbb{N}_{0}\right)$ ). Now, according to equation (8) we have $\Psi^{-1}\left(f^{m}\right)=\operatorname{mred}_{q}\left(F^{m}\right)=\operatorname{mred}_{q}(0)=0$ and consequently $\Psi^{-1}\left(f^{r}\right)=0 \forall r \geq m$. Thus, $f^{r}=1 \forall r \geq m$.

If, on the other hand, there is an $m \in \mathbb{N}$ such that $f^{m+\lambda}=f^{m}=1 \forall \lambda \in \mathbb{N}$ applying the isomorphism $\Psi^{-1}$ (see Remark 3) we obtain $F^{\cdot(m+\lambda)}=F^{m}=0 \forall \lambda \in \mathbb{N}$ and (see equation (8)) $\operatorname{mred}_{q}\left(F^{m+\lambda}\right)=\operatorname{mred}_{q}\left(F^{m}\right)=0 \forall \lambda \in \mathbb{N}$. It follows from equation (7) (see also [5, Remark 47]) that $F^{m+\alpha}=0 \forall \alpha \in \mathbb{N}_{0}$.

Now, by Theorem 2 , there are no sequences $a \rightarrow_{s} b$ between any two arbitrary vertices $a, b$ of length larger than $m-1$. As a conse- 
quence, there cannot be any nontrivial strongly connected components in the dependency graph of $f$.

Definition 12. A monomial dynamical system $f \in M F_{n}^{n}\left(\mathbf{F}_{q}\right)$ whose dependency graph contains nontrivial strongly connected components is called a coupled monomial dynamical system.

Definition 13. Let $G=\left(V_{G}, E_{G}, \pi_{G}\right)$ be a digraph, $m \in \mathbb{N}$ a natural number, and $a, b \in V_{G}$ two vertices. The number of different sequences of length $m$ from $a$ to $b$ is denoted by $s_{m}(a, b) \in \mathbb{N}_{0}$. By Theorem 2 it obviously holds that $s_{m}\left(a_{i}, a_{j}\right)=\left(A^{m}\right)_{i j}$.

Theorem 4. Let $\mathrm{F}_{q}$ be a finite field, $f \in M F_{n}^{n}\left(\mathrm{~F}_{q}\right)$ a coupled monomial dynamical system, and $G_{f}=\left(V_{f}, E_{f}, \pi_{f}\right)$ its dependency graph. Then $f$ is a fixed point system if and only if there is an $m \in \mathbb{N}$ such that the following two conditions hold.

1. For every pair of nodes $a, b \in V_{f}$ with $a \rightarrow m b$ there exists for every $\lambda \in \mathbb{N}$ an $a_{\lambda} \in \mathbb{Z}$ such that $s_{m+\lambda}(a, b)=s_{m}(a, b)+a_{\lambda}(q-1) \neq 0$.

2. For every pair of nodes $a, b \in V_{f}$ with $s_{m}(a, b)=0$ it holds that $s_{m+\lambda}(a, b)=0 \forall \lambda \in \mathbb{N}$.

Proof. Let $V_{f}=\left\{a_{1}, \ldots, a_{n}\right\}$ be the enumeration of the vertices. If $f$ is a fixed point system, $\exists m \in \mathbb{N}$ such that $f^{m+\lambda}=f^{m} \forall \lambda \in \mathbb{N}$. By applying the homomorphism $\Psi^{-1}$ we get (see Remark 3)

$$
F^{(m+\lambda)}=F^{m} \forall \lambda \in \mathbb{N} .
$$

By Remark 5 it follows that $\operatorname{mred}_{q}\left(F^{m+\lambda}\right)=\operatorname{mred}_{q}\left(F^{m}\right) \forall \lambda \in \mathbb{N}$. Let $i, j \in\{1, \ldots, n\}$. If, on the one hand, $\left(F^{m}\right)_{i j}=0$, then by equation (9) we would have $\left(F^{\cdot(m+\lambda)}\right)_{i j}=0 \forall \lambda \in \mathbb{N}$. Consequently, by equation (4) we have $\left(F^{m+\alpha}\right)_{i j}=0 \forall \alpha \in \mathbb{N}_{0}$.

Now, by Theorem 2, there are no sequences $a_{i} \rightarrow_{s} a_{j}$ of length larger than $m-1$. In other words, Theorem 4 condition 2 follows. If, on the other hand, $\left(F^{m}\right)_{i j} \neq 0$ then by equation (9) we would have $\left(F^{(m+\lambda)}\right)_{i j}=\left(F^{m}\right)_{i j} \neq 0 \forall \lambda \in \mathbb{N}$. Consequently, by equations (4) and (6) $\exists a_{\lambda} \in \mathbb{Z}$ such that $\left(F^{m+\lambda}\right)_{i j}=\left(F^{m}\right)_{i j}+a_{\lambda}(q-1) \forall \lambda \in \mathbb{N}$. In other words, Theorem 4 condition 1 follows.

We now show the converse: Given conditions 1 and 2 and in accordance with Theorem 2 and Remark 5, if $\left(F^{m}\right)_{i j}=0$, then $\left(F^{m+\lambda}\right)_{i j}=\left(F^{m}\right)_{i j} \forall \lambda \in \mathbb{N}$ and if $\left(F^{m}\right)_{i j} \neq 0$, then $\exists a_{\lambda} \in \mathbb{Z}$ : $\left(F^{m+\lambda}\right)_{i j}=\left(F^{m}\right)_{i j}+a_{\lambda}(q-1) \neq 0 \forall \lambda \in \mathbb{N}$. 
Now, by equations (4) and (6), we have $\operatorname{mred}_{q}\left(F^{m+\lambda}\right)=$ $\operatorname{mred}_{q}\left(F^{m}\right) \forall \lambda \in \mathbb{N}$ and by Remark $5 F^{\cdot(m+\lambda)}=F^{\cdot m} \forall \lambda \in \mathbb{N}$. Thus, after applying the isomorphism $\Psi: f^{m+\lambda}=f^{m} \forall \lambda \in \mathbb{N}$.

The following parameter for digraphs was introduced into the field of research on monomial dynamical systems over $\mathrm{F}_{2}$ in [8] as the "loop number". The loop number of a strongly connected graph is also known as the "index of imprimitivity" [23] or "period" [24] and has been used in the study of non-negative matrices [25, 26]. It is well known that this number quantizes the length of any closed sequence in a strongly connected digraph. It is also the biggest possible "quantum".

Definition 14. Let $G=\left(V_{G}, E_{G}, \pi_{G}\right)$ be a digraph and $a \in V_{G}$ one of its vertices. The number

$$
L(a):=\min _{\substack{a \rightarrow u \\ a \rightarrow v \\ a \rightarrow v \\ u \neq v}}|u-v|
$$

is called the loop number of $a$. If there is no sequence of positive length from $a$ to $a$, then $\mathcal{L}(a)$ is set to zero.

If $\stackrel{\leftrightarrow}{a}$ is nontrivial, then for every $b \in \stackrel{\leftrightarrow}{a}$ it holds that $\mathcal{L}(b)=\mathcal{L}(a)$ (for a proof see [8, Lemma 4.2]). Therefore, we introduce the loop number of strongly connected components as $\mathcal{L}(\stackrel{\leftrightarrow}{a}):=\mathcal{L}(a)$.

In [25, Section 3.4], the index of imprimitivity is directly defined (i.e., without defining it for single nodes in the digraph) for strongly connected digraphs using an equivalent definition.

Remark 6. The loop number of any trivial strongly connected component is, due to the convention made in Definition 14, equal to zero.

Corollary 1. Let $\mathbf{F}_{q}$ be a finite field, $f \in M F_{n}^{n}\left(\mathbf{F}_{q}\right)$ a coupled monomial dynamical system, and $G_{f}=\left(V_{f}, E_{f}, \pi_{f}\right)$ its dependency graph. If $f$ is a fixed point system, then the loop number of each of its nontrivial strongly connected components is equal to one.

Proof. Let $m \in \mathbb{N}$ be as stated in Theorem 4. Let $\stackrel{\leftrightarrow}{\subseteq} V_{f}$ be a nontrivial strongly connected component. For every $b \in \stackrel{\leftrightarrow}{a}$ we have that $b$ is strongly connected with itself. Therefore, for every $s \in \mathbb{N}$ there is a $t \geq s$ such that $b \rightarrow_{t} b$. In particular, there must be a $u \in \mathbb{N}$ with $u>m$ such that $b \rightarrow_{u} b$, that is, $s_{u}(b, b) \geq 1$. By condition 2 of Theorem 4 we know that $s_{m}(b, b) \neq 0$, otherwise $s_{u}(b, b)=0$. Now, from condition 1 we know that $\exists a_{\lambda} \in \mathbb{Z}$ : $s_{m+\lambda}(b, b)=s_{m}(b, b)+a_{\lambda}(q-1) \neq 0 \forall \lambda \in \mathbb{N}$ and, in particular, 
$s_{m+\lambda}(b, b) \neq 0 \forall \lambda \in \mathbb{N}$. Therefore, $\forall \lambda \in \mathbb{N}$ there are sequences $b \rightarrow_{m+\lambda} b$. Thus, $\mathcal{L}(\stackrel{\leftrightarrow}{a})=\mathcal{L}(b)=1$.

Definition 15. Let $G=\left(V_{G}, E_{G}, \pi_{G}\right)$ be a digraph and $a, b \in V_{G}$ two vertices. The vertex $a$ is called recurrently connected to $b$, if for every $s \in \mathbb{N}$ there is a $u \geq s$ such that $a \rightarrow_{u} b$.

Remark 7. Let $G=\left(V_{G}, E_{G}, \pi_{G}\right)$ be a digraph with vertex set $V_{G}$ of cardinality $n:=\left|V_{G}\right|$. Obviously, for any two vertices $a, b \in V_{G}$ either $a$ is recurrently connected to $b$ or there is an $m \in \mathbb{N}$ with $m \leq n$ such that no sequence $a \rightarrow_{t} b$ of length $t \geq m$ exists.

Lemma 1. Let $G=\left(V_{G}, E_{G}, \pi_{G}\right)$ be a digraph and $U \subseteq V_{G}$ a nontrivial strongly connected component. Furthermore, let $t:=L(U)$ be the loop number of $U$. Then for each $a, b \in U$ there is an $m \in \mathbb{N}$ such that the graph $G$ contains sequences $a \rightarrow_{m+\lambda t} b$ of length $m+\lambda t \forall \lambda \in \mathbb{N}$.

Proof. See [8, Proposition 4.5] or, alternatively, [25, Lemma 3.4.1].

Theorem 5. Let $G=\left(V_{G}, E_{G}, \pi_{G}\right)$ be a digraph containing nontrivial strongly connected components. If the loop number of every nontrivial strongly connected component is equal to one, then there is an $m \in \mathbb{N}$ such that any pair of vertices $a_{i}, a_{j} \in V_{G}$ with $a_{i}$ recurrently connected to $a_{j}$ satisfies $s_{m+\lambda}\left(a_{i}, a_{j}\right)>0 \forall \lambda \in \mathbb{N}_{0}$.

Proof. This follows easily from Lemma 1. Alternatively, see [25, Lemma 3.4.1].

Definition 16. Let $\mathbf{F}_{q}$ be a finite field, $f \in M F_{n}^{n}\left(\mathbf{F}_{q}\right)$ a monomial dynamical system, and $G_{f}=\left(V_{f}, E_{f}, \pi_{f}\right)$ its dependency graph. $f$ is called a $(q-1)$-fold redundant monomial system if there is an $N \in \mathbb{N}$ such that for any pair $a, b \in V_{f}$ with $a$ recurrently connected to $b$, the following holds:

$$
\forall m \geq N \exists \alpha_{a b m} \in \mathbb{N}_{0}: s_{m}(a, b)=\alpha_{a b m}(q-1) .
$$

Remark 8. Note that any Boolean monomial dynamical system $f \in M F_{n}^{n}\left(\mathrm{~F}_{2}\right)$ is $(2-1)$-fold redundant.

Lemma 2. Let $\mathbf{F}_{q}$ be a finite field, $f \in M F_{n}^{n}\left(\mathbf{F}_{q}\right)$ a coupled $(q-1)$-fold redundant monomial dynamical system, and $G_{f}=\left(V_{f}, E_{f}, \pi_{f}\right)$ its dependency graph. Then $f$ is a fixed point system if the loop number of each nontrivial strongly connected component of $G_{f}$ is equal to one.

Proof. Let $V_{f}=\left\{a_{1}, \ldots, a_{n}\right\}$ be the enumeration of the vertices and $F:=\Psi^{-1}(f)$ be the corresponding matrix of $f$. Consider two arbitrary 
vertices $a_{i}, a_{j} \in V_{f}$. By Remark 7, either $a_{i}$ is recurrently connected to $a_{j}$ or there is an $m_{0} \in \mathbb{N}$ with $m_{0} \leq n$ such that no sequence $a \rightarrow_{t} b$ of length $t \geq m_{0}$ exists. If the latter is the case, then $\left(F^{m_{0}+\lambda}\right)_{i j}=0 \forall \lambda \in \mathbb{N}_{0}$.

On the other hand, if $a_{i}$ is recurrently connected to $a_{j}$, then by Theorem 5 there is an $m_{1} \in \mathbb{N}$ such that

$$
s_{m_{1}+\gamma}\left(a_{i}, a_{j}\right)>0 \forall \gamma \in \mathbb{N}_{0} .
$$

Consider now $m_{2}:=\max \left(n, m_{1}\right)$. Due to the universality of $m_{1}$ in equation (10), for any pair of vertices $a_{i}, a_{j} \in V_{G}$ with $a_{i}$ recurrently connected to $a_{j}$ there is a sequence $a_{i} \rightarrow m_{2}+\gamma a_{j}$ of length $m_{2}+\gamma$, in particular $s_{\left(m_{2}+\gamma\right)}\left(a_{i}, a_{j}\right)>0 \forall \gamma \in \mathbb{N}_{0}$. Now, let $N$ be the constant in Definition 16 and $m_{3}:=\max \left(N, m_{2}\right)$. Now, by hypothesis, $\exists \alpha_{i j \gamma} \in \mathbb{N}$ such that $s_{\left(m_{3}+\gamma\right)}\left(a_{i}, a_{j}\right)=\alpha_{i j \gamma}(q-1) \forall \gamma \in \mathbb{N}_{0}$. Thus,

$$
\begin{aligned}
& s_{\left(m_{3}+\gamma\right)}\left(a_{i}, a_{j}\right)= \\
& \alpha_{i j \gamma}(q-1)=\alpha_{i j 0}(q-1)+\left(\alpha_{i j \gamma}-\alpha_{i j 0}\right)(q-1)= \\
& s_{m_{3}}\left(a_{i}, a_{j}\right)+\left(\alpha_{i j \gamma}-\alpha_{i j 0}\right)(q-1) \forall \gamma \in \mathbb{N}_{0} .
\end{aligned}
$$

Summarizing, since $m_{0} \leq n \leq m_{2} \leq m_{3}$, we can say that $\forall i, j \in\{1, \ldots, n\}$, depending on whether $a_{i}$ and $a_{j}$ are recurrently connected or not, $\left(F^{m_{3}+\lambda}\right)_{i j}=0 \forall \lambda \in \mathbb{N}_{0}$ or $\exists a_{\lambda} \in \mathbb{Z}:\left(F^{m_{3}+\lambda}\right)_{i j}=$ $\left(F^{m_{3}}\right)_{i j}+a_{\lambda}(q-1) \neq 0 \forall \lambda \in \mathbb{N}_{0}$. Now, by equations (4) and (6), it follows that $\operatorname{mred}_{q}\left(F^{m_{3}+\lambda}\right)=\operatorname{mred}_{q}\left(F^{m_{3}}\right) \forall \lambda \in \mathbb{N}$ and by Remark 5 $F^{\cdot\left(m_{3}+\lambda\right)}=F^{m_{3}} \forall \lambda \in \mathbb{N}$. Thus, after applying the isomorphism $\Psi$ we have $f^{m_{3}+\lambda}=f^{m_{3}} \forall \lambda \in \mathbb{N}$.

Theorem 6. Let $\mathbf{F}_{q}$ be a finite field, $f \in M F_{n}^{n}\left(\mathbf{F}_{q}\right)$ a coupled ( $\left.q-1\right)$-fold redundant monomial dynamical system, and $G_{f}=\left(V_{f}, E_{f}, \pi_{f}\right)$ its dependency graph. Then $f$ is a fixed point system if and only if the loop number of each nontrivial strongly connected component of $G_{f}$ is equal to one.

Proof. The claim follows immediately from Lemma 2 and Corollary 1.

Theorem 7. Let $\mathrm{F}_{q}$ be a finite field, $f \in M F_{n}^{n}\left(\mathbf{F}_{q}\right)$ a coupled monomial dynamical system, and $G_{f}=\left(V_{f}, E_{f}, \pi_{f}\right)$ its dependency graph. Then $f$ is a fixed point system if the following properties hold. 
1. The loop number of each nontrivial strongly connected component of $G_{f}$ is equal to one.

2. For each nontrivial strongly connected component $\stackrel{\leftrightarrow}{\subseteq} V_{f}$ and arbitrary $b, c \in \stackrel{\leftrightarrow}{a}, s_{1}(b, c) \neq 0 \Rightarrow s_{1}(b, c)=q-1$.

Proof. Let $V_{f}=\left\{a_{1}, \ldots, a_{n}\right\}$ be the enumeration of the vertices. Consider two vertices $a_{i}, a_{j} \in V_{f}$ such that $a_{i}$ is recurrently connected to $a_{j}$. Then by Theorem 5 there is an $m_{1} \in \mathbb{N}$ such that

$$
s_{m_{1}+\gamma}\left(a_{i}, a_{j}\right)>0 \forall \gamma \in \mathbb{N}_{0} .
$$

Consider now $m_{2}:=\max \left(n, m_{1}\right)$. Due to the universality of $m_{1}$ in equation (11), for any pair of vertices $a_{i}, a_{j} \in V_{G}$ with $a_{i}$ recurrently connected to $a_{j}$ there is a sequence $a_{i} \rightarrow m_{2}+\gamma a_{j}$ of length $m_{2}+\gamma$. Since $m_{2}+\gamma>n-1$, necessarily $\exists a_{k_{\gamma}}, a_{l_{\gamma}} \in \overleftrightarrow{a_{k_{\gamma}}}$ such that $\overleftrightarrow{a_{k_{\gamma}}}$ is nontrivial and

$$
a_{i} \rightarrow{ }_{\left(m_{2}+\gamma\right)} a_{j}=a_{i} \rightarrow \cdots \rightarrow a_{k_{\gamma}} \rightarrow_{t} a_{l_{\gamma}} \rightarrow \cdots \rightarrow a_{j}
$$

( $t$ depends on $i, j$, and $\gamma$ ). Now, by hypothesis, every two directly connected vertices $a, b \in \overleftrightarrow{a_{k_{\gamma}}}$ are directly connected by exactly $q-1$ directed edges. Therefore, for any sequence $a_{k_{\gamma}} \rightarrow{ }_{t} a_{l_{\gamma}}$ of length $t \in \mathbb{N}$ there are $(q-1)^{t}$ different copies of it and we can conclude $\exists \alpha \in \mathbb{N}$ such that $s_{t}\left(a_{k_{\gamma}}, a_{l_{\gamma}}\right)=\alpha(q-1)$. As a consequence, there are $\alpha(q-1)$ different copies of the sequence in equation (12). Since we are dealing with an arbitrary sequence $a_{i} \rightarrow_{\left(m_{2}+\gamma\right)} a_{j}$ of fixed length $m_{2}+\gamma, \gamma \in \mathbb{N}_{0}$ we can conclude that $\exists \alpha_{i j \gamma} \in \mathbb{N}$ such that $s_{\left(m_{2}+\gamma\right)}\left(a_{i}, a_{j}\right)=$ $\alpha_{i j \gamma}(q-1) \forall \gamma \in \mathbb{N}_{0}$. Thus, $f$ is a coupled $(q-1)$-fold redundant monomial dynamical system and the claim follows from Lemma 2.

Corollary 2. Let $\mathbf{F}_{2}$ be the finite field with two elements, $f \in M F_{n}^{n}\left(\mathbf{F}_{2}\right)$ a Boolean monomial dynamical system, and $F:=\Psi^{-1}(f) \in M\left(n \times n ; E_{2}\right)$ its corresponding matrix. Furthermore, let $\mathrm{F}_{q}$ be a finite field and $g \in M F_{n}^{n}\left(\mathrm{~F}_{q}\right)$ the monomial dynamical system whose corresponding matrix $G:=\Psi^{-1}(g) \in M\left(n \times n ; E_{q}\right)$ satisfies $\forall i, j \in\{1, \ldots, n\}$

$$
G_{i j}=\left\{\begin{array}{c}
q-1 \text { if } F_{i j}=1 \\
0 \text { if } F_{i j}=0
\end{array}\right.
$$

If $f$ is a fixed point system then $g$ is a fixed point system too. 
Proof. Let $G_{f}=\left(V_{f}, E_{f}, \pi_{f}\right)$ be the dependency graph of $f$. By the definition of $g$, one can easily see that the dependency graph $G_{g}=\left(V_{g}, E_{g}, \pi_{g}\right)$ of $g$ can be generated from $G_{f}$ by adding $q-2$ identical parallel edges for every existing edge. Obviously $G_{f}$ and $G_{g}$ have the same strongly connected components. If $G_{f}$ does not contain any nontrivial strongly connected components, then $G_{g}$ would not contain any either and by Theorem $3 \mathrm{~g}$ would be a fixed point system. If, on the other hand, $G_{f}$ does contain nontrivial strongly connected components, then by [8, Theorem 6.1] each of those components would have loop number 1. From the definition of $g$ it also follows for any pair of vertices $a, b \in E_{g}$ that $s_{1}(a, b) \neq 0 \Rightarrow s_{1}(a, b)=q-1$. By Theorem $7 \mathrm{~g}$ would be a fixed point system.

Example 1. Let $\mathrm{F}_{2}$ be the Boolean finite field and $f \in M F_{n}^{n}\left(\mathrm{~F}_{2}\right)$ the Boolean coupled monomial dynamical system defined by

$$
\begin{aligned}
& f_{1}(x)=x_{1}^{a_{11}} \\
& f_{i}(x)=\left(\prod_{j=1}^{i} x_{j}^{a_{i j}}\right), i=2, \ldots, n
\end{aligned}
$$

where $a_{i j} \in\{0,1\}, i=1, \ldots, n, j=1, \ldots, i$. According to [8, Corollary 6.3], such a system is always a fixed point system. Now, let $\mathbf{F}_{q}$ be a finite field and consider $g \in M F_{n}^{n}\left(\mathbf{F}_{q}\right)$, the coupled monomial dynamical system defined by

$$
\begin{aligned}
& g_{1}(x)=x_{1}^{\mu\left(a_{11}\right)} \\
& g_{i}(x)=\left(\prod_{j=1}^{i} x_{j}^{\mu\left(a_{i j}\right)}\right), i=2, \ldots, n
\end{aligned}
$$

where

$$
\mu(a):=\left\{\begin{array}{c}
q-1 \text { if } a=1 \\
0 \text { if } a=0 .
\end{array}\right.
$$

Such a system is called triangular. By Corollary 2, $g$ must be a fixed point system.

Theorem 8. Let $\mathrm{F}_{q}$ be a finite field, $f \in M F_{n}^{n}\left(\mathrm{~F}_{q}\right)$ a coupled monomial dynamical system, and $G_{f}=\left(V_{f}, E_{f}, \pi_{f}\right)$ its dependency graph. Then $f$ is a fixed point system if for every vertex $a \in V_{f}$ that is recurrently 
connected to some other vertex $b \in V_{f}$ the edge $a \rightarrow a$ appears exactly $q-1$ times in $E_{f}$, that is, $\left|\pi_{f}^{-1}((a, a))\right|=q-1$.

Proof. Let $V_{f}=\left\{a_{1}, \ldots, a_{n}\right\}$ be the enumeration of the vertices and $F:=\Psi^{-1}(f)$ be the corresponding matrix of $f$. Consider two vertices $a_{i}, a_{j} \in V_{f}$ such that $a_{i}$ is recurrently connected to $a_{j}$. Then by Theorem 5 there is an $m_{1} \in \mathbb{N}$ such that

$$
s_{m_{1}+\gamma}\left(a_{i}, a_{j}\right)>0 \forall \gamma \in \mathbb{N}_{0} .
$$

Consider now $m_{2}:=\max \left(n, m_{1}\right)$. Due to the universality of $m_{1}$ in equation (13), for any pair of vertices $a_{i}, a_{j} \in V_{G}$ with $a_{i}$ recurrently connected to $a_{j}$ there is a sequence $a_{i} \rightarrow m_{2}+\gamma a_{j}$ of length $m_{2}+\gamma$. Consider one particular sequence $a_{i} \rightarrow m_{2}+\gamma a_{j}$ of length $m_{2}+\gamma$ and call it $w_{\gamma}:=a_{i} \rightarrow_{m_{2}+\gamma} a_{j}$. By hypothesis there are exactly $q-1$ directed edges $a_{i} \rightarrow a_{i}$. Therefore, there are $q-1$ copies of the sequence $w_{\gamma}$. Since we are dealing with an arbitrary sequence $a_{i} \rightarrow\left(m_{2}+\gamma\right) a_{j}$ of fixed length $m_{2}+\gamma, \quad \gamma \in \mathbb{N}_{0}$ we can conclude that $\exists \alpha_{i j \gamma} \in \mathbb{N}$ such that $s_{\left(m_{2}+\gamma\right)}\left(a_{i}, a_{j}\right)=\alpha_{i j \gamma}(q-1) \forall \gamma \in \mathbb{N}_{0}$. Thus, $f$ is a coupled $(q-1)$ fold redundant monomial dynamical system and the claim follows from Lemma 2.

Example 2. Let $\mathbf{F}_{q}$ be a finite field and $f \in M F_{n}^{n}\left(\mathbf{F}_{q}\right)$ a monomial dynamical system such that the diagonal entries of its corresponding matrix $F:=\Psi^{-1}(f)$ satisfy $F_{i i}=q-1 \forall i \in\{1, \ldots, n\}$. Since every vertex satisfies the requirement of Theorem $8, f$ must be a fixed point system.

\section{An Algorithm of Polynomial Complexity to Identify Fixed Point} Systems

\subsection{Some Basic Considerations}

Definition 17. Let $X$ be a nonempty finite set, $n \in \mathbb{N}$ a natural number, and $f: X^{n} \rightarrow X^{n}$ a time discrete finite dynamical system. The phase space of $f$ is the digraph with node set $X^{n}$, arrow set $E$ defined as $E:=\left\{(x, y) \in X^{n} \times X^{n} \mid f(x)=y\right\}$, and vertex mapping

$$
\begin{aligned}
\pi & : E \rightarrow X^{n} \times X^{n} \\
(x, y) & \mapsto(x, y) .
\end{aligned}
$$

According to our definition of monomial dynamical system $f \in M F_{n}^{n}\left(\mathbf{F}_{q}\right)$, the possibility that one of the functions $f_{i}$ is equal to 
the zero function is excluded (see Definition 3 and Remark 1). Therefore, the following algorithm is designed for such systems. However, in this algorithmic framework it would be convenient to include the more general case (as defined in $[8,13]$ ), that is, the case when some of the functions $f_{i}$ can indeed be equal to the zero function. In the vein of Remark 1 this actually only requires some type of preprocessing. The preprocessing algorithm is described and analyzed in [5, Appendix B].

Our algorithm is based on the following observation made by Dr. Michael Shapiro about general time discrete finite dynamical systems: a chain of transient states in the phase space of a time discrete finite dynamical system $f: X^{n} \rightarrow X^{n}$ can contain at most $s:=\left|X^{n}\right|-1=|X|^{n}-1$ transient elements. Therefore, to determine whether a system is a fixed point system, it is sufficient to establish whether the mappings $f^{r}$ and $f^{r+1}$ are identical for any $r \geq s$. In the case of a monomial system $f \in M F_{n}^{n}\left(\mathrm{~F}_{q}\right)$, due to the isomorphism of the monoids $M\left(n \times n ; E_{q}\right)$ and $M F_{n}^{n}\left(\mathbf{F}_{q}\right)$ (see Section 2), we only need to look at the corresponding matrices $F^{r}, F^{r+1} \in M\left(n \times n ; E_{q}\right)$ (see also [27] in the Boolean case). Computationally, it is more convenient to generate the following sequence of powers:

$$
F^{2},\left(F^{\cdot 2}\right)^{\cdot 2}=F^{4},\left(F^{4}\right)^{\cdot 2}=F^{\cdot 8},\left(F^{8}\right)^{\cdot 2}=F^{\cdot 16}, \ldots, F^{\cdot\left(2^{t}\right)} .
$$

To achieve the "safe" number of iterations $\left|\mathrm{F}_{q}^{n}\right|-1=q^{n}-1$ we need to make sure $2^{t} \geq q^{n}-1$. This is equivalent to $t \geq \log _{2}\left(q^{n}-1\right)$. To obtain a natural number we use the ceil function:

$$
t:=\operatorname{ceil}\left(\log _{2}\left(q^{n}-1\right)\right) \text {. }
$$

Thus we have, due to the monotonicity of the log function, $t<\log _{2}\left(q^{n}-1\right)+1 \leq \log _{2}\left(q^{n}\right)+1=n \log _{2}(q)+1$.

In the Boolean case, the studies performed in [27] on upper bounds for the length of the transient part of the sequence $\left(F^{i}\right)_{i \in \mathbb{N}}$ of consecutive powers of $F$ could significantly improve our algorithm. Such bounds could provide us with a better (i.e., lower) lower bound than equation (14) for $t$. It would be beyond the scope of this article to elaborate on these aspects of the Boolean case.

\subsection{The Algorithm and Its Complexity Analysis}

The algorithm is fairly simple. Given a monomial system $f \in M F_{n}^{n}\left(\mathrm{~F}_{q}\right)$ and its corresponding matrix $F:=\Psi^{-1}(f) \in M\left(n \times n ; E_{q}\right)$ :

1. With $t$ as defined by equation (14), calculate the matrices $A:=F^{\cdot 2^{t}}$ and $B:=$ FA. This step requires $t+1$ matrix multiplications. 
2. Compare the $n^{2}$ entries $A_{i j}$ and $B_{i j}$. This step requires at most $n^{2}$ comparisons. (This maximal value is needed in the case that $f$ is a fixed point system.)

3. $f$ is a fixed point system if and only if the matrices $A$ and $B$ are equal.

It is well known that matrix multiplication requires $2 n^{3}-n^{2}$ addition or multiplication operations. Since $t+1<n \log _{2}(q)+2$, the number of operations required in step 1 is bounded above by $\left(2 n^{3}-n^{2}\right)\left(n \log _{2}(q)+2\right)$. Summarizing, we have the following upper bound $N(n, q)$ for the number of operations in steps 1 and 2:

$$
N(n, q):=\left(2 n^{3}-n^{2}\right)\left(n \log _{2}(q)+2\right)+n^{2} .
$$

For a fixed size $q$ of the finite field $\mathrm{F}_{q}$ used it holds that

$$
\lim _{n \rightarrow \infty} \frac{N(n, q)}{n^{4}}=2 \log _{2}(q)
$$

and we can conclude $N(n, q) \in O\left(n^{4}\right)$ for a fixed $q$. The asymptotic behavior for a growing number of variables and growing number of field elements is described by

$$
\lim _{\substack{n \rightarrow \infty \\ q \rightarrow \infty}} \frac{N(n, q)}{n^{4} \log _{2}(q)}=2 .
$$

Thus, $N(n, q) \in \mathrm{O}\left(n^{4} \log _{2}(q)\right)$ for $n, q \rightarrow \infty$.

It is necessary to comment on the arithmetic operations performed during the matrix multiplications. Since the matrices are elements of the matrix monoid $M\left(n \times n ; E_{q}\right)$, the arithmetic operations are operations in the monoid $E_{q}$. The addition (resp., multiplication) operation on $E_{q}$ requires an integer number addition (resp., multiplication) and a reduction using $\operatorname{red}_{q}$ (see Section 2). For a detailed description of integer number representation and arithmetic in typical computer algebra systems see [28, Chapter 4].

The reduction $\operatorname{red}_{q}(a)$ of an integer number $a \in \mathbb{N}_{0}, a \geq q$ is obtained as the degree of the remainder of the polynomial division $\tau^{a} \div\left(\tau^{q}-\tau\right)$. According to [28, Section 4.6.5] this division requires $\mathrm{O}\left(2\left(\operatorname{deg}\left(\tau^{a}\right)-\operatorname{deg}\left(\tau^{q}-\tau\right)\right)\right)=\mathrm{O}(2(a-q))$ integer number operations. However, we know that the reductions $\operatorname{red}_{q}($.$) are applied to the re-$ sult of (regular integer) addition or multiplication of elements of $E_{q}$ and therefore 


$$
a-q \leq\left\{\begin{array}{c}
2(q-1)-q=q-2 \\
(q-1)^{2}-q=q^{2}-q+1
\end{array}\right.
$$

As a consequence, in the worst case scenario, one addition (resp., multiplication) in the monoid $E_{q}$ requires $O(q)$ (resp., $O\left(q^{2}\right)$ ) regular integer number operations.

Since $E_{q}$ is a finite set and only the results of $n^{2}$ pairwise additions and $n^{2}$ pairwise multiplications are needed, the numbers are stored in a table after the first time they are calculated while the algorithm is running. Since most of the commercial and freely available computer algebra systems provide implementations of finite field arithmetic and arithmetic of polynomials over a finite ring, the implementation of our algorithm is a very simple task. We have successfully implemented the algorithm in Maple ${ }^{\mathrm{TM}}$. The code can be made available from the author upon request.

Since the matrix multiplications dominate the complexity of the algorithm, for very large systems more efficient matrix multiplication algorithms could be used. Indeed, using Strassen's algorithm [29, 30], a complexity of $O\left(n^{\omega+1} \log _{2}(q)\right)$, where $\omega \leq \log _{2}(7) \approx 2.807$ could be reached. The performance could be substantially improved through the use of parallelization techniques [31, 32].

It is pertinent to mention that while this article was being peerreviewed, the article [14] was published, in which an algorithm of complexity $\mathrm{O}\left(n^{3} \log _{2}\left(n \log _{2}(q)\right)\right.$ is presented, that is used to determine whether an $n$-dimensional linear dynamical system $L: R^{n} \rightarrow R^{n}$ over a finite $\operatorname{ring} R$ of cardinality $q=|R|$ is a fixed point system. While the authors of [14] did perform a complexity analysis of their algorithm, they did not elaborate on the details of a concrete computer implementation of the arithmetic operations on the finite ring $R$ and thus, did not provide information on the computational cost of such operations.

In [13] it is stated that in order to exploit their results algorithmically, an algorithm would be required to determine whether or not a linear dynamical system $L: R^{n} \rightarrow R^{n}$ over a finite ring $R$ is a fixed point system. The algorithm presented in [14] provides this missing ingredient. Nevertheless, for a given $f \in M F_{n}^{n}\left(\mathbf{F}_{q}\right)$, prior to being able to apply the algorithm from [14], a linear dynamical system $L(f):(\mathbb{Z} /(q-1))^{n} \rightarrow(\mathbb{Z} /(q-1))^{n}$ and a Boolean monomial dynamical system $T(f) \in M F_{n}^{n}\left(\mathrm{~F}_{2}\right)$ need to be constructed (see [13] for the details). The overall computational complexity of an algorithm exploiting the results of [13] and [14] still remains to be investigated. 


\section{Acknowledgments}

We would like to thank Dr. Omar Colón-Reyes for his hospitality and a very fruitful academic interaction at the University of Puerto Rico, Mayagüez. We are grateful to Dr. Bodo Pareigis for offering the opportunity to participate in a great seminar at the Ludwig-Maximilians-Universität in Munich, Germany. We also would like to express our gratitude to Dr. Michael Shapiro for very helpful comments. We are grateful to Dr. Karen Duca for her support and to Dr. David Thorley-Lawson for providing an excellent research environment at the Pathology Department of Tufts University, the institute where the material for this paper was conceived. Moreover, we thank Dr. Jill Roughan, Dr. Michael Shapiro and Dr. David Thorley-Lawson for proofreading the manuscript.

The author acknowledges support by a Public Health Research Grant (RO1 AI062989) to Dr. David Thorley-Lawson at Tufts University, Boston, MA, USA. The author was affiliated with Centre for Mathematical Sciences, Munich University of Technology, Boltzmannstr.3, 85747 Garching, Germany and also with Pathology Department, Tufts University, 150 Harrison Ave., Boston, MA 02111, USA during the development of the material presented here.

\section{References}

[1] A. W. Burks, ed., Essays on Cellular Automata, Urbana, IL: University of Illinois Press, 1970.

[2] J. Reger and K. Schmidt, "Modeling and Analyzing Finite State Automata in the Finite Field $F_{2}$," Mathematics and Computers in Simulation, 66(2-3), 2004 pp. 193-206. doi .1016/j.matcom.2003.11.005.

[3] C. L. Barrett, H. S. Mortveit, and C. M. Reidys, "Elements of a Theory of Simulation II: Sequential Dynamical Systems," Applied Mathematics and Computation, 107(2-3), 2000 pp. 121-136. doi.10.1016/S0096-3003(98)10114-5.

[4] R. Lidl and H. Niederreiter, Finite Fields, 2nd ed., Vol. 20 (Encyclopedia of Mathematics and Its Applications), New York: Cambridge University Press, 1997 (foreword by P. M. Cohn).

[5] E. Delgado-Eckert, "Monomial Dynamical and Control Systems over a Finite Field and Applications to Agent-Based Models in Immunology," Ph.D. thesis, Munich, Germany: Technische Universität München, 2008. http://mediatum2.ub.tum.de/doc/645326/document.pdf.

[6] J. Kari, "Theory of Cellular Automata: A Survey," Theoretical Computer Science, 334(1-3), 2005 pp. 3-33. doi.10.1016/j.tcs.2004.11.021.

[7] R. Bartlett and M. Garzon, "Monomial Cellular Automata," Complex Systems, 7(5), 1993 pp. 367-388.

[8] O. Colón-Reyes, R. Laubenbacher, and B. Pareigis, "Boolean Monomial Dynamical Systems," Annals of Combinatorics, 8(4), 2004 pp. 425-439. doi.10.1007/s00026-004-0230-6.

Complex Systems, 18 ㄷ 2009 Complex Systems Publications, Inc. 
[9] A. Khrennikov and M. Nilsson, "On the Number of Cycles of $p$-adic Dynamical Systems," Journal of Number Theory, 90(2), 2001 pp. 255-264. doi.10.1006/jnth.2001.2665.

[10] M. Nilsson, "Fuzzy Cycles of $p$-adic Monomial Dynamical Systems," Far East Journal of Dynamical Systems, 5(2), 2003 pp. 149-173.

[11] T. Vasiga and J. Shallit, "On the Iteration of Certain Quadratic Maps over GF(p),” Discrete Mathematics, 277(1-3), 2004 pp. 219-240.

[12] O. Colón-Reyes, Monomial Dynamical Systems over Finite Fields, Ph.D. thesis, Blacksburg, VA: Virginia Tech., 2005.

[13] O. Colón-Reyes, A. S. Jarrah, R. Laubenbacher, and B. Sturmfels, "Monomial Dynamical Systems over Finite Fields," Complex Systems, 16(4), 2006 pp. 333-342.

[14] G. Xu and Y. M. Zou, "Linear Dynamical Systems over Finite Rings," Journal of Algebra, 321(8), 2009 pp. 2149-2155.

[15] E. Delgado-Eckert, "Boolean Monomial Control Systems," Mathematical and Computer Modelling of Dynamical Systems, 15(2), 2009 pp. 107-137. doi.10.1080/13873950902808594.

[16] B. Elspas, "The Theory of Autonomous Linear Sequential Networks," IRE Transactions on Circuit Theory, 6(1), 1959 pp. 45-60.

[17] R. A. Hernández Toledo, "Linear Finite Dynamical Systems," Communications in Algebra, 33(9), 2005 pp. 2977-2989. doi.10.1081/AGB-200066211.

[18] J. Reger and K. Schmidt, "A Finite Field Framework for Modeling, Analysis and Control of Finite State Automata," Mathematical and Computer Modelling of Dynamical Systems (MCMDS), 10(3-4), 2004 pp. 253-285. doi.10.1080/13873950412331300142.

[19] D. K. Milligan and M. J. D. Wilson, "The Behavior of Affine Boolean Sequential Networks," Connection Science, 5(2), 1993 pp. 153-167.

[20] P. Cull, "Linear Analysis of Switching Nets," Biological Cybernetics, 8(1), 1971 pp. 31-39. doi.10.1007/BF00270831.

[21] W. Just, "The Steady State System Problem Is NP-Hard Even for Monotone Quadratic Boolean Dynamical Systems.” (Jun 3, 2006)

www.math.ohiou.edu/ just/PAPERS/monNPh14.pdf.

[22] F. Harary, R. Z. Norman, and D. Cartwright, Structural Models: An Introduction to the Theory of Directed Graphs, New York: Wiley \& Sons, 1965.

[23] V. Pták and I. Sedlaček, "On the Index of Imprimitivity of Nonnegative Matrices," Czechoslovak Mathematical Journal, 8(83), 1958 pp. 496-501.

[24] E. V. Denardo, "Periods of Connected Networks and Powers of Nonnegative Matrices," Mathematics of Operations Research, 2(1), 1977 pp. 20-24. doi.10.1287/moor.2.1.20.

[25] R. A. Brualdi and H. J. Ryser, Combinatorial Matrix Theory, Vol. 39 (Encyclopedia of Mathematics and Its Applications), New York: Cambridge University Press, 1991.

[26] P. Lancaster and M. Tismenetsky, The Theory of Matrices: With Applications, 2nd ed., (Computer Science and Applied Mathematics). Orlando, FL: Academic Press Inc., 1985. 
[27] B. De Schutter and B. De Moor, "On the Sequence of Consecutive Powers of a Matrix in a Boolean Algebra," SIAM Journal on Matrix Analysis and Applications, 21(1), 1999 pp. 328-354.

doi.10.1137/S0895479897326079.

[28] M. Kaplan, Computeralgebra, Berlin: Springer-Verlag, 2004.

[29] V. Strassen, "Gaussian Elimination Is Not Optimal," Numerische Mathematik, 13(4), 1969 pp. 354-356.

[30] D. H. Bailey, K. Lee, and H. Simon, “Using Strassen's Algorithm to Accelerate the Solution of Linear Systems," The Journal of Supercomputing, 4(4), 1991 pp. 357-371. doi .10.1007/BF00129836.

[31] M. Ben-Ari, Principles of Concurrent and Distributed Programming: Algorithms and Models, 2nd ed., New York: Addison-Wesley, 2006.

[32] A. Pollard, D. J. K. Mewhort, and D. F. Weaver, eds., High Performance Computing Systems and Applications, New York: SpringerVerlag, 2000. 